\title{
A note on likelihood ratio ordering between parallel systems with two exponential components
}

\author{
Emanuel Emanouilidis ${ }^{\mathrm{a}}$, Jiantian Wang ${ }^{\mathrm{b}, *}$ \\ a School of Computer Science, Kean University, Union, NJ, 07083, USA. \\ ${ }^{b}$ School of Mathematical Science, Kean University, Union, NJ, 07083, USA.
}

\begin{abstract}
With the aid of computer programming, we obtain a result on stochastic comparison of the lifetime of two parallel systems with two exponential components in terms of likelihood ratio ordering. This result reveals a more comprehensive picture on stochastic ordering between parallel systems and thus provides a relatively satisfied answer to an open problem raised in [N. Balakrishnan, P. Zhao, Probab. Engrg. Inform. Sci., 27 (2013), 403-443].
\end{abstract}

Keywords: Parallel system, stochastic comparison, likelihood ratio order.

2010 MSC: 90B25, 60E15.

(C)2019 All rights reserved.

\section{Introduction}

To enhance the reliability of a device, parallel systems are commonly used. Therefore, to compare the lifetimes of parallel systems is fundamental in reliability theory, as well as in many other fields, such as, statistical inference, operations research, and applied probability.

The lifetimes of parallel systems can be described by the largest statistics. The research on stochastic comparison for the largest statistics has a long history. As so far, many interesting results have been established. See, for instance, $[2,4-6,10,11]$. One also may refer to the book $[8,9]$, and a nice survey article of [1].

However, due to technical issue, the study in this field is far from comprehensive, even for the simplest case where the parallel systems consist of two exponential components. The comparison of two parallel systems with exponential components is basic and critical to the stochastic comparison of general k-outof- $n$ systems. Also, it is related with some other problems, such as, redundancy allocation problems, see, [7].

For $i=1,2$, let $X_{i}$ be an exponential random variable with hazard rate $\lambda_{i}$, and $Y_{i}$ be an exponential random variable with hazard rate $\mu_{i}$. Let $X_{2: 2}=\max \left\{X_{1}, X_{2}\right\}$ and $Y_{2: 2}=\max \left\{Y_{1}, Y_{2}\right\}$. Denote $X_{2: 2}$ as $T\left(\lambda_{1}, \lambda_{2}\right)$ and $Y_{2: 2}$ as $T\left(\mu_{1}, \mu_{2}\right)$. In the sequel, we assume $\lambda_{1} \leqslant \lambda_{2}$ and $\mu_{1} \leqslant \mu_{2}$.

\footnotetext{
${ }^{*}$ Corresponding author

Email addresses: eemanoui@kean.edu (Emanuel Emanouilidis), jwang@kean.edu (Jiantian Wang)

doi: $10.22436 /$ jmcs.019.04.05
}

Received: 2019-01-02 Revised: 2019-05-30 Accepted: 2019-06-23 
To compare $T\left(\lambda_{1}, \lambda_{2}\right)$ and $T\left(\mu_{1}, \mu_{2}\right)$, Dykstra et al. (1997) showed that if $\left(\lambda_{1}, \lambda_{2}\right) \stackrel{m}{\succ}\left(\mu_{1}, \mu_{2}\right), T\left(\lambda_{1}, \lambda_{2}\right) \geqslant_{\operatorname{lr}}$ $\mathrm{T}\left(\mu_{1}, \mu_{2}\right)$, here $\stackrel{\mathrm{m}}{\succ}$ stands for majorization order. [11] revealed that when $\lambda_{1} \leqslant \mu_{1} \leqslant \mu_{2} \leqslant \lambda_{2}$ and $\lambda_{1}+$ $\lambda_{2} \leqslant \mu_{1}+\mu_{2}, T\left(\lambda_{1}, \lambda_{2}\right) \geqslant \operatorname{lr} T\left(\mu_{1}, \mu_{2}\right)$. [10] showed, when $\lambda_{1} \leqslant \mu_{1} \leqslant \lambda_{2} \leqslant \mu_{2}$ and $\lambda_{1}+\mu_{2} \leqslant \mu_{1}+\lambda_{2}$, $\mathrm{T}\left(\lambda_{1}, \lambda_{2}\right) \geqslant \operatorname{lr} \mathrm{T}\left(\mu_{1}, \mu_{2}\right)$.

Let $\mathfrak{u}=\left(\mu_{1}, \mu_{2}\right)-\left(\lambda_{1}, \lambda_{2}\right)$. The above results can be combined as, when $\mathbf{u}=(1,-1)+\mathbf{b}(0,1)$, with $0 \leqslant b \leqslant 2$,

$$
\mathrm{T}\left(\lambda_{1}, \lambda_{2}\right) \geqslant_{\operatorname{lr}} \mathrm{T}\left(\mu_{1}, \mu_{2}\right)
$$

By extensive empirical check, [1] observed that to get the above results, the sufficient conditions are somewhat stringent. They posted an open problem on whether there are sharper sufficient conditions for the result (1).

With the aid of computer programming, we prove that $b$ can be extended to $0 \leqslant b \leqslant 6$. Such a result provides a relatively satisfied answer to an open problem posed in [1].

\section{Definitions and notations}

Let $X$ be a nonnegative continuous random variable with distribution function $F_{X}(t)$, survival function $S_{X}(t)=1-F_{X}(t)$, and density function $f_{X}(t)$. The hazard function and the reversed hazard function of $X$ are defined as $\lambda_{X}=f_{X} / S_{X}$ and $r_{X}=f_{X} / F_{X}$, respectively. For two nonnegative continuous random variables $X$ and $Y$, we say $X$ is larger than $Y$ in the usual stochastic order (denoted by $X \geqslant_{s t} Y$ ), if $S_{X}(t) \geqslant S_{Y}(t) ; X$ is larger than $Y$ in hazard rate order (denoted by $X \geqslant_{h r} Y$ ), if $\lambda_{X}(t) \leqslant \lambda_{Y}(t) ; X$ is larger than $Y$ in reversed hazard rate order (denoted by $X \geqslant r_{r h} Y$ ), if $r_{X}(t) \geqslant r_{Y}(t)$; and $X$ is larger than $Y$ in likelihood ratio order (denoted by $X \geqslant_{\operatorname{lr}} Y$ ), if the ratio $f_{X}(t) / f_{Y}(t)$ is increasing in $t$. It is well-known that the likelihood ratio order implies several other orders, such as, the usual stochastic order, the hazard rate order, and the reversed hazard rate order.

Given two vectors $\mathbf{a}=\left(a_{1}, a_{2}, \ldots, a_{n}\right)$ and $\mathbf{b}=\left(b_{1}, b_{2}, \ldots, b_{n}\right)$ with increasing elements, the vector $\mathbf{a}$ is said to majorize the vector $\mathbf{b}$ (denoted as $\mathbf{a} \stackrel{\mathrm{m}}{\succ} \mathbf{b}$ ) if, $\sum_{i=1}^{n} a_{i}=\sum_{i=1}^{n} b_{i}$, and $\sum_{i=1}^{k} a_{i} \leqslant \sum_{i=1}^{k} b_{i}$, for $k=1, \ldots, n-1$. For two vectors $\mathbf{a}$ and $\mathbf{b}$, if there is a positive constant $k$, such that, $\mathbf{a}=k \mathbf{b}$, we then say these two vectors are equal, and simply denote by $\mathbf{a}=\mathbf{b}$.

\section{Main result and proof}

Theorem 3.1. Let $\mathbf{u}=(1,-1)+\mathbf{b}(0,1), 0 \leqslant \mathrm{~b} \leqslant 6$, then, when $\left(\mu_{1}, \mu_{2}\right)-\left(\lambda_{1}, \lambda_{2}\right)=\mathbf{u}$,

$$
\mathrm{T}\left(\lambda_{1}, \lambda_{2}\right) \geqslant_{\operatorname{lr}} \mathrm{T}\left(\mu_{1}, \mu_{2}\right)
$$

Proof. The density function of $X=T\left(\lambda_{1}, \lambda_{2}\right)$ is

$$
f_{\lambda}(t)=\lambda_{1} e^{-\lambda_{1} t}+\lambda_{2} e^{-\lambda_{2} t}-\left(\lambda_{1}+\lambda_{2}\right) e^{-\left(\lambda_{1}+\lambda_{2}\right) t},
$$

and that of $Y$ is

$$
g_{\mu}(t)=\mu_{1} e^{-\mu_{1} t}+\mu_{2} e^{-\mu_{2} t}-\left(\mu_{1}+\mu_{2}\right) e^{-\left(\mu_{1}+\mu_{2}\right) t} .
$$

The condition $f_{\lambda}(t) / g_{\mu}(t)$ is increasing in $t>0$ is equivalent to

$$
f_{\lambda}^{\prime}(t) g_{\mu}(t)-f_{\lambda}(t) g_{\mu}^{\prime}(t) \geqslant 0,
$$

which is equivalent to

$$
\frac{f_{\lambda}^{\prime}(t)}{f_{\lambda}(t)}-\frac{g_{\mu}^{\prime}(t)}{g_{\lambda}(t)} \geqslant 0 .
$$


We have,

$$
\frac{f_{\lambda}^{\prime}(t)}{f_{\lambda}(t)}=-\frac{\lambda_{1}^{2} e^{-\lambda_{1} t}+\lambda_{2}^{2} e^{-\lambda_{2} t}-\left(\lambda_{1}+\lambda_{2}\right)^{2} e^{-\left(\lambda_{1}+\lambda_{2}\right) t}}{\lambda_{1} e^{-\lambda_{1} t}+\lambda_{2} e^{-\lambda_{2} t}-\left(\lambda_{1}+\lambda_{2}\right) e^{-\left(\lambda_{1}+\lambda_{2}\right) t}}=-\frac{\lambda_{1}^{2} e^{\lambda_{2} t}+\lambda_{2}^{2} e^{\lambda_{1} t}-\left(\lambda_{1}+\lambda_{2}\right)^{2}}{\lambda_{1} e^{\lambda_{2} t}+\lambda_{2} e^{\lambda_{1} t}-\left(\lambda_{1}+\lambda_{2}\right)} .
$$

Consider function

$$
\Phi\left(x_{1}, x_{2}\right)=\frac{x_{1}^{2} e^{x_{2}}+x_{2}^{2} e^{x_{1}}-\left(x_{1}+x_{2}\right)^{2}}{x_{1} e^{x_{2}}+x_{2} e^{x_{1}}-\left(x_{1}+x_{2}\right)}, \quad 0<x_{1} \leqslant x_{2} .
$$

It is clear that, if $\Phi\left(x_{1}, x_{2}\right)$ is increasing in the direction $\mathbf{u}$, then, $T\left(\lambda_{1}, \lambda_{2}\right) \geqslant_{\operatorname{lr}} T\left(\mu_{1}, \mu_{2}\right)$. Therefore, we just need to show $\Phi\left(x_{1}, x_{2}\right)$ is increasing in $u$. The result of [3] indicated $\Phi\left(x_{1}, x_{2}\right)$ is increasing in $(1,-1)$, so, we only need to check the direction $(1,5)$.

Let $b(x)=\left(e^{x}-1\right) / x$, then,

$$
\Phi\left(x_{1}, x_{2}\right)=\frac{x_{2} b\left(x_{1}\right)+x_{1} b\left(x_{2}\right)-2}{b\left(x_{1}\right)+b\left(x_{2}\right)}
$$

For two numbers $A$ and $B$, if their signs are the same, we denote them as $A \stackrel{\text { sgn }}{=} B$. By some simple calculus, we obtain

$$
\begin{aligned}
\nabla(1,5) \Phi= & \frac{\partial \Phi}{\partial x_{1}}+5 \frac{\partial \Phi}{\partial x_{2}} \\
\stackrel{\text { sgn }}{=} & {\left[x_{2} b^{\prime}\left(x_{1}\right)+b\left(x_{2}\right)+5 b\left(x_{1}\right)+5 x_{1} b^{\prime}\left(x_{2}\right)\right] \times\left[b\left(x_{1}\right)+b\left(x_{2}\right)\right] } \\
& -\left[x_{2} b\left(x_{1}\right)+x_{1} b\left(x_{2}\right)-2\right]\left[b^{\prime}\left(x_{1}\right)+5 b^{\prime}\left(x_{2}\right)\right] \\
= & \left(x_{2}-x_{1}\right) b^{\prime}\left(x_{1}\right) b\left(x_{2}\right)+6 b\left(x_{1}\right) b\left(x_{2}\right)+b^{2}\left(x_{2}\right) \\
& +5 b^{2}\left(x_{1}\right)-5\left(x_{2}-x_{1}\right) b\left(x_{1}\right) b^{\prime}\left(x_{2}\right)+2\left[b^{\prime}\left(x_{1}\right)+5 b^{\prime}\left(x_{2}\right)\right] \\
\geqslant & 6 b\left(x_{1}\right) b\left(x_{2}\right)+b^{2}\left(x_{2}\right)-5\left(x_{2}-x_{1}\right) b\left(x_{1}\right) b^{\prime}\left(x_{2}\right) .
\end{aligned}
$$

Consider the function $I(x, y)=6 b\left(x_{1}\right) b\left(x_{2}\right)+b^{2}(y)-5(y-x) b(x) b^{\prime}(y)$ with $0<x \leqslant y$. We have

$$
\begin{aligned}
I(x, y) & =6 b(x) b(y)+b^{2}(y)-5(y-x) b(x) b^{\prime}(y) \\
& \stackrel{\text { sgn }}{=} 6 b(x) y\left(e^{y}-1\right)+\left(e^{y}-1\right)^{2}-5(y-x) b(x)\left[(y-1) e^{y}+1\right] \\
& =e^{2 y}+e^{y}[6 b(x) y-5 b(x)(y-x)(y-1)-2]+1-6 b(x) y-5 b(x)(y-x) .
\end{aligned}
$$

Let $y=x+t$, then,

$$
\begin{aligned}
I(x, y) & =e^{2 x+2 t}+e^{x+t}[6 b(x)(x+t)-2-5 b(x) t(x+t-1)]+1-6 b(x)(x+t)-5 b(x) t \\
& \stackrel{s g n}{=} e^{2 t}+e^{t}\left[\alpha_{2}(x) t^{2}+\alpha_{1}(x) t+\alpha_{0}(x)\right]+\beta_{1}(x) t+\beta_{0}(x) \triangleq I(t),
\end{aligned}
$$

where $\alpha_{2}(x)=-5 b(x) / e^{x}, \alpha_{1}(x)=[11 b(x)-5 x b(x)] / e^{x}, \alpha_{0}=[6 x b(x)-2] / e^{x}, \beta_{1}(x)=-11 b(x) / e^{2 x}$, and $\beta_{0}(x)=[1-6 x b(x)] / e^{2 x}$. We have

$$
\begin{aligned}
I^{\prime}(t) & =2 e^{2 t}+e^{t}\left[\alpha_{2} t^{2}+\left(2 \alpha_{2}+\alpha_{1}\right) t+\left(\alpha_{1}+\alpha_{0}\right)\right]+\beta_{1}, \\
I^{\prime \prime}(t) & =4 e^{2 t}+e^{t}\left[\alpha_{2} t^{2}+\left(4 \alpha_{2}+\alpha_{1}\right) t+\left(2 \alpha_{2}+2 \alpha_{1}+\alpha_{0}\right)\right] \\
& \stackrel{\operatorname{sgn}}{=} 4 e^{t}+\alpha_{2} t^{2}+\left(4 \alpha_{2}+\alpha_{1}\right) t+\left(2 \alpha_{2}+2 \alpha_{1}+\alpha_{0}\right) \\
& \geqslant\left(\alpha_{2}+2\right) t^{2}+\left(4 \alpha_{2}+\alpha_{1}+4\right) t+\left(2 \alpha_{2}+2 \alpha_{1}+\alpha_{0}+4\right) \triangleq J(t) .
\end{aligned}
$$

By Lemma .1 (in Appendix), we know, $\mathrm{J}(0)=2 \alpha_{2}+2 \alpha_{1}+\alpha_{0}+4 \geqslant 0$, and $\mathrm{J}(1)=7 \alpha_{2}+3 \alpha_{1}+\alpha_{0}+10 \geqslant$ 0 . When $\alpha_{2}+2 \leqslant 0$, it is clear that $J(t) \geqslant 0$ for $0 \leqslant t \leqslant 1$. When $\alpha_{2}+2 \geqslant 0$, we also have $J(t) \geqslant 0$ for $0 \leqslant t \leqslant 1$. If not, then the equation $J(t)=0$ has two solutions $t_{1}, t_{2}$ in $0 \leqslant t \leqslant 1$. So

$$
0<-\frac{4 \alpha_{2}+\alpha_{1}+4}{2\left(\alpha_{2}+2\right)}<1
$$


that is, $6 \alpha_{2}+\alpha_{1}>0$. But, as we show in Lemma .1, it is impossible. Therefore, we obtain that, for $0 \leqslant t \leqslant 1, J(t) \geqslant 0$, and hence, $I^{\prime \prime}(t) \geqslant 0$ for $0 \leqslant t \leqslant 1$.

Replace $t$ by $t+1$ in $I^{\prime \prime}(t)$. We have

$$
\begin{aligned}
I^{\prime \prime}(1+t) & \stackrel{\text { sgn }}{=} 4 e^{1+t}+\alpha_{2}(1+t)^{2}+\left(4 \alpha_{2}+\alpha_{1}\right)(1+t)+\left(2 \alpha_{2}+2 \alpha_{1}+\alpha_{0}\right) \\
& \stackrel{\text { sgn }}{=} e^{t}+\alpha_{2}^{\prime} t^{2}+\alpha_{1}^{\prime} t+\alpha_{0}^{\prime} \\
& \geqslant 1+t+\frac{t^{2}}{2}+\frac{t^{3}}{6}+\alpha_{2}^{\prime} t^{2}+\alpha_{1}^{\prime} t+\alpha_{0}^{\prime} \\
& \stackrel{\operatorname{sgn}}{=} t^{3}+\left(3+6 \alpha_{2}^{\prime}\right) t^{2}+6\left(1+\alpha_{1}^{\prime}\right) t+6\left(1+\alpha_{0}^{\prime}\right) \triangleq \mathrm{K}(\mathrm{t}),
\end{aligned}
$$

where $\alpha_{2}^{\prime}=\alpha_{2} /(4 e), \alpha_{1}^{\prime}=\left(6 \alpha_{2}+\alpha_{1}\right) /(4 e), \alpha_{0}^{\prime}=\left(7 \alpha_{2}+3 \alpha_{1}+\alpha_{0}\right) /(4 e)$.

By Lemma .2 (in Appendix), $3+6 \alpha_{2}^{\prime} \geqslant 0,1+\alpha_{0}^{\prime} \geqslant 0$, and for $x \geqslant 3,1+\alpha_{1}^{\prime} \geqslant 0$. Thus, for $x \geqslant 3$, $K(t) \geqslant 0$.

By Lemma .1, in $x \geqslant 0$, both of the functions $\mathrm{I}_{2}(x)=3+6 \alpha_{2}^{\prime}(x)$ and $\mathrm{I}_{1}(x)=6\left[1+\alpha_{1}^{\prime}(x)\right]$ are increasing, while the function $\mathrm{I}_{0}(x)=6\left[1+\alpha_{0}^{\prime}(x)\right]$ is decreasing. Hence, for $2.5 \leqslant x \leqslant 3$,

$$
K(t)=t^{3}+I_{2}(x) t^{2}+I_{1}(x) t+I_{0}(x) \geqslant t^{3}+I_{2}(2.5) t^{2}+I_{1}(2.5) t+I_{0}(3) \geqslant t^{3}+1.9 t^{2}-0.4 t+0.9 \triangleq \theta_{1}(t) .
$$

For $0 \leqslant x \leqslant 2.5,1+\alpha_{1}^{\prime}<0$. Let $a=-\left(1+\alpha_{0}^{\prime}\right) /\left(1+\alpha_{1}^{\prime}\right)$. Then, when $0 \leqslant t \leqslant a, K(t) \geqslant 0$. By Lemma $.2,0 \leqslant x \leqslant 2.5, a \geqslant 0.7$. Thus, for $0 \leqslant t \leqslant 0.7, k(t) \geqslant 0$.

Replace $t$ by $t+0.7$, we have

$$
\begin{aligned}
\mathrm{K}(\mathrm{t}+0.7) & =(\mathrm{t}+0.7)^{3}+\left(3+6 \alpha_{2}^{\prime}\right)(\mathrm{t}+0.7)^{2}+6\left(1+\alpha_{1}^{\prime}\right)(\mathrm{t}+0.7)+6\left(1+\alpha_{0}^{\prime}\right) \\
& \geqslant \mathrm{t}^{3}+\left(5.1+6 \alpha_{2}^{\prime}\right) \mathrm{t}^{2}+\left[1.4\left(3+6 \alpha_{2}^{\prime}\right)+6\left(1+\alpha_{1}^{\prime}\right)+1.47\right] \mathrm{t}+0.49\left(3+6 \alpha_{2}^{\prime}\right)+0.343 .
\end{aligned}
$$

By Lemma .2 , for $x \geqslant 1,1.4\left(3+6 \alpha_{2}^{\prime}\right)+6\left(1+\alpha_{1}^{\prime}\right)+1.47>0$. Hence, for $1 \leqslant x \leqslant 2.5, k(t) \geqslant 0$.

For $0.5 \leqslant x \leqslant 1$

$$
K(t)=t^{3}+I_{2}(x) t^{2}+I_{1}(x) t+I_{0}(x) \geqslant t^{3}+I_{2}(0.5) t^{2}+I_{1}(0.5) t+I_{0}(1.0) \geqslant t^{3}+0.82 t^{2}-3.4 t+1.75 \triangleq \theta_{2}(t) .
$$

For $0.25 \leqslant x \leqslant 0.5$

$$
K(t)=t^{3}+I_{2}(x) t^{2}+I_{1}(x) t+I_{0}(x) \geqslant t^{3}+I_{2}(0.25) t^{2}+I_{1}(0.25) t+I_{0}(0.5) \geqslant t^{3}+0.55 t^{2}-3.9 t+2.5 \triangleq \theta_{3}(t) .
$$

For $0.1 \leqslant x \leqslant 0.25$

$$
K(t)=t^{3}+I_{2}(x) t^{2}+I_{1}(x) t+I_{0}(x) \geqslant t^{3}+I_{2}(0.1) t^{2}+I_{1}(0.1) t+I_{0}(0.25) \geqslant t^{3}+0.37 t^{2}-4.3 t+3 \triangleq \theta_{4}(t) .
$$

For $0 \leqslant x \leqslant 0.1$

$$
K(t)=t^{3}+I_{2}(x) t^{2}+I_{1}(x) t+I_{0}(x) \geqslant t^{3}+I_{2}\left(0^{+}\right) t^{2}+I_{1}\left(0^{+}\right) t+I_{0}(0.1) \geqslant t^{3}+0.24 t^{2}-4.5 t+3.47 \triangleq \theta_{5}(t) .
$$

By Lemma .3 (in Appendix), for $i=1, \ldots, 5, \theta_{i}(t) \geqslant 0$. Thus, we show $K(t) \geqslant 0$, and hence, $I^{\prime \prime}(t) \geqslant 0$.

From Lemma .1, we know, $\mathrm{I}^{\prime}(0)=2+\alpha_{1}+\alpha_{0}+\beta_{1} \geqslant 0$, and $\mathrm{I}(0)=1+\alpha_{0}++\beta_{0} \geqslant 0$. Therefore, we arrive at the conclusion of $I(t) \geqslant 0$. The theorem is thus proved.

\section{Discussion}

Comparing the lifetime of two parallel systems with exponential components is the basic and simplest case in stochastic comparison of two general k-out-of-n systems. However, even for this simplest situation, the investigation is not so comprehensive. [1] gave some examples showing $T(2.05,8) \geqslant \operatorname{lr} T(4,11)$, 
$\mathrm{T}(2,4.5) \geqslant_{\operatorname{lr}} \mathrm{T}(4,6)$, but $\mathrm{T}(2,4.5) \ngtr \operatorname{lr} \mathrm{T}(2.05,8)$. We have, $\mathfrak{u}_{1}=(4,11)-(2.05,8)=(1.95,3)=(1,1.54)$, $\mathbf{u}_{2}=(4,6)-(2,4.5)=(1,0.75)$, and $\mathbf{u}_{3}=(2.05,8)-(2,4.5)=(1,70)$. By Theorem 3.1, in $\mathfrak{u}_{1}, \mathfrak{u}_{2}$, the likelihood ratio order exists. These examples coincide with Theorem 3.1.

While in direction $\mathfrak{u}_{3}=(1,70)$, their example shows that there is no likelihood ratio order in that direction. It brings an interesting question, that is, which is the smallest number $\delta$, such that, in the direction $(1, \delta)$, there is no likelihood ratio order?

From our computer simulations, we find $\delta$ can be 12. Thus, we can conclude that, in the direction $\mathfrak{u}=(1, \delta)$, when $-1 \leqslant \delta \leqslant 5$, there is likelihood ratio order; while for $\delta>12$, there is no likelihood ratio order. For the cases when $5 \leqslant b \leqslant 12$, the conclusion is not so clear. All these need further investigations.

For technical simplicity, in this paper, we just focus on the case of exponential components. We believe that the idea and the method can be applied to other components, such as Weibull components.

\section{Appendix A}

Lemma .1. Let $b(x)=\left(e^{x}-1\right) / x, \alpha_{2}(x)=-5 b(x) / e^{x}, \alpha_{1}(x)=[11 b(x)-5 x b(x)] / e^{x}, \alpha_{0}(x)=[6 x b(x)-$ $2] / e^{x}, \beta_{1}(x)=-11 b(x) / e^{2 x}$, and $\beta_{0}(x)=[1-6 x b(x)] / e^{2 x}$. Then, for $x \geqslant 0$, we have

$$
\begin{aligned}
& \mathrm{I}_{1}(x)=2 \alpha_{2}(x)+2 \alpha_{1}(x)+\alpha_{0}(x)+4 \geqslant 0, \\
& I_{2}(x)=7 \alpha_{2}(x)+3 \alpha_{1}(x)+\alpha_{0}(x)+10 \geqslant 0, \\
& I_{3}(x)=6 \alpha_{2}(x)+\alpha_{1}(x)<0, \\
& I_{4}(x)=2+\alpha_{1}(x)+\alpha_{0}(x)+\beta_{1}(x) \geqslant 0, \\
& I_{5}(x)=1+\alpha_{0}(x)+\beta_{0}(x) \geqslant 0 .
\end{aligned}
$$

Proof. The proofs for these inequalities are quite similar. So, we just check a few. We have

$$
\begin{aligned}
& I_{1}(x)=2 \alpha_{2}(x)+2 \alpha_{1}(x)+\alpha_{0}(x)+4 \\
& \stackrel{\text { sgn }}{=}-10 b(x)+2[11 b(x)-5 x b(x)]+6 x b(x)-2+4 e^{x} \\
& =12 \mathrm{~b}(\mathrm{x})+2>0 \text {, } \\
& \mathrm{I}_{4}(\mathrm{x})=2+\alpha_{1}(\mathrm{x})+\alpha_{0}(\mathrm{x})+\beta_{1}(\mathrm{x}) \\
& \stackrel{\text { sgn }}{=} 2 e^{2 x}+11 e^{x} b(x)+x e^{x} b(x)-2 e^{x}-11 b(x) \\
& \stackrel{\operatorname{sgn}}{=} 2 x e^{2 x}+11 e^{x}\left(e^{x}-1\right)+x e^{x}\left(e^{x}-1\right)-2 x e^{x}-11\left(e^{x}-1\right) \\
& =2 x e^{x}\left(e^{x}-1\right)+11\left(e^{x}-1\right)^{2}+x e^{x}\left(e^{x}-1\right) \geqslant 0 .
\end{aligned}
$$

Lemma .2. From the functions mentioned in Lemma .1, define $\alpha_{2}^{\prime}(x)=\alpha_{2}(x) /(4 e), \alpha_{1}^{\prime}(x)=\left[6 \alpha_{2}(x)+\alpha_{1}(x)\right] /(4 e)$, $\alpha_{0}^{\prime}(x)=\left[7 \alpha_{2}(x)+3 \alpha_{1}(x)+\alpha_{0}(x)\right] /(4 e)$. Let

$$
\begin{aligned}
& \mathrm{J}_{1}(\mathrm{x})=3+6 \alpha_{2}^{\prime}(\mathrm{x}), \\
& \mathrm{J}_{2}(\mathrm{x})=1+\alpha_{0}^{\prime}(\mathrm{x}), \\
& \mathrm{J}_{3}(\mathrm{x})=1+\alpha_{1}^{\prime}(\mathrm{x}), \\
& \mathrm{J}_{4}(\mathrm{x})=1.4\left[3+6 \alpha_{2}^{\prime}(\mathrm{x})\right]+6\left[1+\alpha_{1}^{\prime}(\mathrm{x})\right]+1.47, \\
& \mathrm{~J}_{5}(\mathrm{x})=-\left[1+\alpha_{0}^{\prime}(\mathrm{x})\right] /\left[1+\alpha_{1}^{\prime}(\mathrm{x})\right] .
\end{aligned}
$$

Then, $\mathrm{J}_{1}(\mathrm{x})$ and $\mathrm{J}_{3}(\mathrm{x})$ are increasing in $\mathrm{x} \geqslant 0$, while the function $\mathrm{J}_{2}(\mathrm{x})$ is decreasing. For $\mathrm{x} \geqslant 0, \mathrm{~J}_{1}(\mathrm{x}) \geqslant 0$, $\mathrm{J}_{2}(\mathrm{x}) \geqslant 0$, and when $\mathrm{x} \geqslant 3, \mathrm{~J}_{3}(\mathrm{x}) \geqslant 0 . \mathrm{J}_{4}(\mathrm{x})$ increasing, and for $\mathrm{x}>1, \mathrm{~J}_{4}(\mathrm{x})>0$. For $0 \leqslant \mathrm{x} \leqslant 2.5, \mathrm{~J}_{5}(\mathrm{x}) \geqslant 0.7$. 
Proof. Because of similarity, we just check the statement about $\mathrm{J}_{5}(x)$. Since $\mathrm{J}_{3}(x)=1+\alpha_{1}^{\prime}(x)$ is increasing, then, for $0 \leqslant x \leqslant 2.5, J_{3}(x) \leqslant J_{3}(2.5)<0$. Thus, $0 \leqslant x \leqslant 2.5$,

$$
\begin{aligned}
\mathrm{J}_{5}(\mathrm{x}) & =-\left[1+\alpha_{0}^{\prime}(\mathrm{x})\right] /\left[1+\alpha_{1}^{\prime}(\mathrm{x})\right]-0.7 \\
& \stackrel{\mathrm{sgn}}{=}-\frac{4 e+7 \alpha_{2}+3 \alpha_{1}+\alpha_{0}}{4 e+6 \alpha_{2}+\alpha_{1}}-0.7 \\
& \stackrel{\operatorname{sgn}}{=}\left[4 e+7 \alpha_{2}+3 \alpha_{1}+\alpha_{0}\right]+0.7\left[4 e+6 \alpha_{2}+\alpha_{1}\right] \\
& =11.2 \alpha_{2}(x)+3.7 \alpha_{1}(x)+\alpha_{0}(x)+6.8 e \\
& \stackrel{\operatorname{sgn}}{=}-11.2 \times 5 b(x)+3.7[11 b(x)-5 x b(x)]+6 x b(x)-2+6.8 e^{x+1} \\
& \geqslant-15.3 b(x)-12.5 x b(x)+18.4 e^{x}-2 \\
& \stackrel{\operatorname{sgn}}{=} 5.9 x e^{x}-15.3\left(e^{x}-1\right)+10.5 x \\
& \stackrel{\operatorname{sgn}}{=} 5.9 e^{x}-15.3 b(x)+10.5 \\
& \triangleq J(x) .
\end{aligned}
$$

We have

$$
\begin{aligned}
J(x) & =5.9 \sum_{k=0}^{\infty} \frac{x^{k}}{k !}-15.3 \sum_{k=0}^{\infty} \frac{x^{k}}{(k+1) !}+10.5 \\
& =\sum_{k=1}^{\infty} \frac{x^{k}}{(k+1) !}[(k+1) \times 5.9-15.3]+1.1 \\
& \geqslant 1.1-1.75 x+0.4 x^{2}+0.3 x^{3} \\
& \triangleq J(x) .
\end{aligned}
$$

We have, $\mathrm{J}^{\prime}(x)=0.9 x^{2}+0.8 x-1.75$. Clearly, $\mathrm{J}^{\prime}(0)<0$ and $\mathrm{J}^{\prime}(1)<0$. Thus, $\mathrm{J}^{\prime}(\mathrm{x})<0$ in $0<x<1$. Thus, $J(x) \geqslant J(1)>0$. Now, replace $x$ by $x+1$, we have

$$
J(x+1)=0.3 x^{3}+1.3 x^{2}-0.45 x+0.05 \geqslant 1.3 x^{2}-0.45 x+0.05 \geqslant 0 .
$$

Lemma .3. Let

$$
\begin{aligned}
& \theta_{1}(t)=t^{3}+1.9 t^{2}-0.4 t+0.9 \\
& \theta_{2}(t)=t^{3}+0.82 t^{2}-3.4 t+1.75 \\
& \theta_{3}(t)=t^{3}+0.55 t^{2}-3.9 t+2.5 \\
& \theta_{4}(t)=t^{3}+0.37 t^{2}-4.3 t+3 \\
& \theta_{5}(t)=t^{3}+0.24 t^{2}-4.5 t+3.47
\end{aligned}
$$

Then, for $\mathrm{t} \geqslant 0, \theta_{i}(t) \geqslant 0$, for $i=1, \ldots, 5$.

Proof. We just show $\theta_{5}(t) \geqslant 0$. Others can be proved in the same way. Clearly, for $0 \leqslant t \leqslant 3.47 / 4.5$, $\theta_{5}(t) \geqslant 0$. So, when $0 \leqslant t \leqslant 0.7, \theta_{5}(t) \geqslant 0$. Replace $t$ by $t+0.7$, we have

$$
\begin{aligned}
\theta_{5}(t+0.7) & =(t+0.7)^{3}+0.24(t+0.7)^{2}-4.5(t+0.7)+3.47 \\
& =t^{3}+2.34 t^{2}-2.694 t+0.7806 \\
& \geqslant 2.34 t^{2}-2.694 t+0.7806 \geqslant 0
\end{aligned}
$$




\section{Acknowledgment}

The authors are grateful to the anonymous referees for their careful proof-reading. Their valuable comments have led to an improved version of this article.

\section{References}

[1] N. Balakrishnan, P. Zhao, Ordering properties of order statistics from heterogeneous populations: a review with an emphasis of some recent developments, Probab. Engrg. Inform. Sci., 27 (2013), 403-443. 1, 4

[2] P. J. Boland, E. El-Neweihi, F. Proschan, Applications of hazard rate ordering in reliability and order statistics, J. Appl. Probab., 31 (1994), 180-192. 1

[3] R. Dykstra, S. Kochar, J. Rojo, Stochastic comparisons of parallel systems of heterogeneous exponential components, J. Statist. Plann. Inference, 65 (1997), 203-211. 3

[4] B. Khaledi, S. C. Kochar, Stochastic orderings among order statistics and sample spacings, in: Uncertainty and optimality, 2002 (2002), 167-203. 1

[5] S. Kochar, J. Rojo, Some new results on stochastic comparisons of spacings from heterogeneous exponential distributions, J. Multivariate Anal., 59 (1996), 272-281.

[6] S. Kochar, M. C. Xu, Stochastic comparisons of parallel systems when components have proportional hazard rates, Probab. Engrg. Inform. Sci., 21 (2007), 597-609. 1

[7] H. Laniado, R. E. Lillo, Allocation policies of redundancies in two-parallelseries and two-seriesparallel systems, IEEE Trans. Reliab., 63 (2014), 223-229. 1

[8] A. Müller, D. Stoyan, Comparison Methods for Stochastic Models and Risks, John Wiley \& Sons, Chichester, (2002). 1

[9] M. Shaked, J. G. Shanthikumar, Stochastic Orders, Springer, New York, (2007). 1

[10] R. F. Yan, G. F. Da, P. Zhao, Further Results for Parallel Systems with Two Heterogeneous Exponential Components, Statistics, 47 (2013), 1128-1140. 1

[11] P. Zhao, N. Balakrishnan, Some characterization results for parallel systems with two heterogeneous exponential components, Statistics, 45 (2011), 593-604. 1 